\title{
Expectativas de los pacientes respecto de la consulta con un médico de familia: estudio de corte transversal
}

\author{
Patient expectations regarding consultation with a family doctor: cross-sectional study
}

Marcos Nusfaumer ${ }^{a}$, Sergio Terrasa $a^{a, b, c}$

\begin{abstract}
Resumen
Antecedentes. El modelo biopsicosocial propone que la atención de la salud se centre en las necesidades de las personas. Sin embargo, no siempre los profesionales de la salud conocen adecuadamente lo que esperan de los encuentros clínicos sus pacientes.

Objetivo. Explorar las expectativas respecto de sus encuentros clínicos de los/as pacientes atendidos por médicos de familia en un Centro de Salud de Atención Primaria del Conurbano Bonaerense (Buenos Aires, Argentina).

Métodos. Estudio observacional descriptivo de corte transversal llevado a cabo sobre una muestra consecutiva de pacientes que acudieron a las consultas médicas de un centro de salud. Se les pidió a los pacientes que de un listado de diez potenciales expectativas, valoraran y priorizaran las más importantes para esa consulta.

Resultados. Fueron entrevistadas 146 personas ( $91 \%$ mujeres) con una mediana de edad de 30 años.

Las expectativas más mencionadas ( $94 \%$ de las personas encuestadas) fueron que el médico demuestre interés y escuche a sus pacientes, y que les brinde explicaciones sobre sus problemas y dudas.

Conclusiones. Hemos podido documentar que las expectativas de nuestros pacientes respecto de sus entrevistas clínicas están muy vinculadas a su componente actitudinal (predisposición a escuchar con interés sus problemas) y al de las habilidades comunicacionales del profesional de la salud (explicaciones).
\end{abstract}

\begin{abstract}
Background. The biopsychosocial model proposes that health care focus on the needs of people. However, health professionals do not always know what their patients expect from clinical meetings.

Objective. To explore the expectations regarding their clinical meetings of the patients attended by family doctors in a Primary Health Care Center of Buenos Aires, Argentina.

Methods. Descriptive cross-sectional observational study carried out on a consecutive sample of patients who attended the medical consultations of a health center. Patients were asked to rate and prioritize the most important ones for that consultation from a list of ten potential expectations.

Results. 146 people ( $91 \%$ women) with a median age of 30 years were interviewed.

The most mentioned expectations ( $94 \%$ of the people surveyed) were for the doctor to show interest and listen to their patients, and to give them explanations about their problems and doubts.

Conclusions. We have been able to document that our patients' expectations regarding their clinical interviews are closely linked to their attitudinal component (predisposition to listen with interest to their problems) and to the communication skills of the healthcare professional (explanations).
\end{abstract}

Palabras clave: Atención Primaria de Salud, Motivación, Medicina Familiar y Comunitaria. Keywords: Primary Health Care, Motivation, Family Practice.

Nusfaumer M, Terrasa S.. Expectativas de los pacientes respecto de la consulta con un médico de familia: estudio de corte transversal. Evid Actual Pract Ambul. 2019;22(4):e002027.

\section{Introducción}

Las expectativas y los valores de los pacientes respecto de los aspectos comunicacionales y operativos de las entrevistas clínicas

En la construcción de la relación entre los profesionales de la salud y las personas a las que atienden, conocer sus valores, sus expectativas, su percepción respecto del curso de los encuentros clínicos, tiene gran relevancia ${ }^{1,2}$, y podemos decir que es inherente a los objetivos de la medicina ${ }^{3}$. Dentro de este conocimiento y con el propósito de mejorar los resultados en cuanto al tratamiento y la satisfacción del/la paciente, se vuelve una necesidad dar espacio y conocer cuáles son las expectativas con respecto a la atención médica que tienen las personas de las comunidades en donde trabajamos ${ }^{4}$.
Desde algunas décadas, el modelo biopsicosocial ${ }^{5}$ propone dentro de sus principios, que la atención médica debe centrarse en las necesidades de las personas, considerando sus conocimientos, creencias y expectativas ${ }^{1}$. Por este motivo, y por otro lado, por las influencias del mercado y la necesidad de mejoras en la gestión, los sistemas de salud han visto necesaria otra relación con el/la paciente que se distancia del modelo paternalista tradicional en miras de mejorar la atención y cumplir con las expectativas de los/as usuarios/as ${ }^{6}$

En este contexto, la medicina familiar interpreta que el profesional debe especializarse en el/la paciente por sobre un cuerpo de conocimientos, enfermedades o procedimientos técnicos ${ }^{1,7}$. Para cumplir con esta premisas, la interacción del profesional con el/la paciente y su familia es una parte muy importante de este proceso $^{7}$.

\footnotetext{
a Servicio de Medicina Familiar y Comunitaria, Hospital Italiano de Buenos Aires. marcosnusfaumer@gmail.com

b Departamento de Investigación, Hospital Italiano de Buenos Aires.

c Departamento de Salud Pública, Instituto Universitario Hospital Italiano de Buenos Aires.
} 
Sin embargo, no siempre los profesionales de la salud logran discernir de manera adecuada lo que espera el/la paciente del encuentro con ellos $/ \mathrm{as}^{8}$. Por ejemplo, la comunicación durante el encuentro clínico puede ser un aspecto a fortalecer y valorar, ya que suele operar como una barrera para obtener buenos resultados en la atención ${ }^{9}$, y existe evidencia que sugiere que algunos resultados en salud se encuentran asociados a la calidad percibida del vínculo entre el/la paciente y el equipo de salud ${ }^{10,11}$.

Por eso, consideramos pertinente que el equipo de salud explore lo que esperan de su trabajo sus usuarios/as. En este contexto retomamos la definición Kravitz ${ }^{11}$, que sostiene que el/la usuario/a de un sistema de salud tiene tres tipos de expectativas: 1) la que logra verbalizar durante el episodio de atención (expectativa explícita); 2) la que no logra verbalizar pero igualmente figura entre sus deseos de cómo deberá transcurrir el encuentro (expectativa implícita); 3) la que representa lo que cree que probablemente va a suceder durante el encuentro clínico (sea o no deseado), basado en sus experiencias previas con el sistema de salud y las experiencias de la comunidad de donde proviene.

En la bibliografía sobre estrategias de mejora continua de la calidad de atención en salud se destaca como un valor para el/la cliente, paciente o ciudadano/ $\mathrm{a}^{12}$, la satisfacción con dicho sistema de cuidados, particularmente en lo referido a lo que los usuarios esperan de las consultas de atención primaria ${ }^{8}$.

\section{La problemática de la evaluación de las expectativas de los usuarios}

Las expectativas respecto de los encuentros clínicos varían en función de los valores y de las experiencias de los/as propios/as usuarios/as, de sus problemas de salud, de las características personales de los proveedores de salud y de sus vínculos con los usuarios/as, y de los marcos institucionales donde estos procesos suceden ${ }^{11}$.

La mayoría de la bibliografía ha abordado esta problemática a nivel institucional. Varios trabajos realizados en España abordaron esta problemática. Por ejemplo, Ruiz Moral et al. ${ }^{8}$ documentaron en una unidad de Medicina Familiar que los aspectos más valorados por las personas atendidas eran los relacionados con la comunicación, lo que no había sido identificado por sus proveedores de salud. A través de una investigación cualitativa por grupos focales, Lapuente et al. ${ }^{13}$ destacaron el valor que le asignan los/as usuarios/as a las cuestiones organizativas del centro de salud; mientras que Redondo Martín et al. ${ }^{4}$ documentaron que las expectativas de los/as pacientes se vinculan al trato que les brindan los/as profesionales y al tiempo dedicado por estos en la consulta ${ }^{4}$. Estos hallazgos concuerdan con los obtenidos en otras investigaciones realizadas en Líbano ${ }^{14}$ y Nigeria ${ }^{15}$. Por otro lado, una investigación realizada en Estonia documentó que los/as pacientes tenían más interés en recibir explicaciones biomédicas de sus dolencias que una atención con foco en lo psicosocial ${ }^{16}$.

Finalmente, los autores de una revisión sistemática ${ }^{6}$ en la que se evaluaron 23 trabajos, incluyendo estudios transversales, cohortes y ensayos aleatorizados, no encontraron asociación entre la percepción de los/as pacientes de que se hubieran cumplido sus expectativas respecto de los encuentros clínicos y resultados "duros" de gestión de salud. Resaltamos que la mayoría de los estudios previos se han enfocado en valorar la satisfacción $\mathrm{del} / \mathrm{la}$ usuario/a tanto con las entrevistas clínicas que han tenido como globalmente, evaluando al centro de salud donde se brinda ala tención primaria ${ }^{17}$.

\section{Justificación de esta investigación}

Consideramos que conocer las expectativas de los/las pacientes respecto a lo que va a ocurrir durante sus consultas médicas permitirá diseñar ciclos de mejora de su calidad en el caso de que se documentara que existe discordancia entre lo que esperan de sus consultas y lo que perciben que reciben.

En el mismo sentido nos acerca al conocimiento de la satisfacción del/la usuario/a como uno de los parámetros importantes para la evaluación de la calidad en atención primaria.

La finalidad del presente trabajo de investigación es explorar las expectativas respecto de sus encuentros clínicos de los/as pacientes que concurren a consultas médicas en un Centro de Salud de Atención Primaria del Conurbano Bonaerense.

\section{Metodología}

\section{Diseño}

Estudio observacional descriptivo de corte transversal.

\section{Población}

Adultos que acudieron a consultas programadas y no programadas al Centro de Salud San Pantaleón (Boulogne, San Isidro, Buenos Aires, Argentina), atendido por residentes de medicina familiar y sus instructores durante Noviembre de 2016, con motivo de realizar una consulta vinculada con su propia salud o la de algún familiar a cargo (p. ej., un niño).

Fueron excluidos aquellos usuarios que realizaron consultas de tipo administrativas, por ejemplo, la solicitud de un certificado de aptitud física para realizar ejercicio, rehacer órdenes o prescripciones vencidas, completar fichas de salud, etc. Para evitar el sesgo de cortesía con el entrevistador, también fueron excluidos los pacientes que lo tenían como médico de cabecera.

\section{Ámbito del estudio}

El barrio "Bajo Boulogne", se encuentra en la localidad argentina de Boulogne Sur Mer del Partido de San Isidro, en la Zona Norte del Conurbano Bonaerense, a $16 \mathrm{Km}$ de la Ciudad Autónoma de Buenos Aires. Según datos del Censo de 2010, el Partido de San Isidro tiene 291.680 habitantes y 75.043 viven en el "Bajo Boulogne". Su población es urbana y se caracteriza por su alta vulnerabilidad sociosanitaria. El barrio cuenta con dos centros asistenciales que brindan sus servicios en forma gratuita: el Centro de Medicina Familiar y Comunitaria San Pantaleón y el Centro de Atención Primaria y Promoción de la Salud Bajo Boulogne ${ }^{18}$.

\section{Variables}

Fueron recolectadas la edad, el sexo y documentado el tipo de consulta (programada vs. no programada) para la cual fueron evaluadas las expectativas de los/las pacientes, y la antigüedad como usuario/a de ese centro de salud.

\section{Recolección de la información}

La información fue recolectada mediante un cuestionario diseñado por el investigador principal local, basado en los resultados comunicados por Ruiz- Moral ${ }^{8}$ que habían sido obtenidos a través de un instrumento autoadministrado, al que no pudimos tener acceso.

Dado que el instrumento original había sido desarrollado en España, y atendiendo a diferencias lingüísticas locales, los investigadores decidimos reemplazar la palabra prescripción por receta y la palabra pruebas por análisis u otros estudios.

El cuestionario fue de tipo autocompletable, con una breve asistencia del entrevistador, y estuvo compuesto por una primera hoja en donde se le solicitaba al paciente que indicara, sobre una lista de diez expectativas (verTabla 1), cómo considera cada una de ellas en una escala ordinal de tres opciones (importante, muy importante o dudoso). En una segunda, hoja el paciente tenía que seleccionar las tres expectativas que consideraba más importantes y también las tres que consideraba menos importantes de esas mismas diez expectativas. 
La validez aparente del cuestionario fue evaluada por un grupo de expertos (tres médicos/as de familia) y se realizó una prueba piloto para corroborar su interpretabilidad por parte de candidatos elegidos en el mismo centro de salud y por parte de empleados administrativos de dicho centro, todos oriundos y habitantes de dicho barrio.

El cuestionario autoadministrable, que contenía un primer párrafo que explicaba los motivos de la investigación a modo de consentimiento verbal, fue entregado por el investigador principal -residente de medicina familiar previamente entrenado- a todas las personas que cumplieron los criterios de elegibilidad que se encontraban en la sala de espera, antes de entrar a su consulta.

Tabla 1. Listado de expectativas propuestas a los usuarios de un Centro de Atención Primaria del Conurbano Bonaerense de Argentina antes de la consulta con un médico de familia. Fuente: modificado de Ruiz-Moral et al. Rev Calid Asist. 2008;23(2):45-51

Expectativas de los pacientes
Que el médico demuestre interés y me escuche
Que tenga suficiente tiempo
Que me ofrezca apoyo y me tranquilice
Que me brinde explicaciones sobre mis problemas y mis dudas
Que me aconseje sobre lo que debo hacer
Que me de un diagnóstico
Que me revise
Que me derive a un especialista
Que me solicite análisis y estudios
Que me haga una receta

\section{Resultados}

Fueron invitadas a participar de la investigación 160 personas. De éstas, 146 aceptaron participar y devolvieron el cuestionario con respuestas. La mediana de edad de los entrevistados fue 30 años (rango intercuartilo [RIC], 24 a 40), $91 \%$ de sexo femenino (131/144). La mayoría de las consultas (100/137; $73 \%)$ fueron programadas. La mediana de tiempo de atención en el Centro de salud fue 2,5 años (RIC, 1 a $6 ; 143$ encuestados).

Todas las expectativas propuestas fueron consideradas como importantes por la mayoría de la población encuestada, destacándose entre las más seleccionadas: "Que el médico demuestre interés y me escuche" $y$ "Que me brinde explicaciones sobre mis problemas y mis dudas" (ambas señaladas por el $94 \%$ de los participantes) y "Que me de un diagnóstico" (90\%). Las expectativas menos seleccionadas fueron "Que me haga una receta" (67\%), "Que me de apoyo y me tranquilice" y "Que tenga suficiente tiempo" (73\% en ambas).
Respecto de la segunda parte del cuestionario, las dos expectativas más jerarquizadas fueron "Que el médico demuestre interés y me escuche" (70,9\%) y "Que me brinde explicaciones sobre mis problemas y mis dudas" (56\%), seguida por "Que me solicite análisis y estudios" (46,2\%). Las expectativas menos valoradas fueron "Que tenga suficiente tiempo" (53,8\%), "Que me haga una receta" (53\%) y "Que me ofrezca apoyo y me tranquilice" (42,7\%).

\section{Discusión}

Nuestros principales hallazgos contradicen nuestros propios prejuicios -probablemente magnificados por sesgos de disponibilidad-, respecto de que una gran parte de nuestros pacientes desearían que se les resolvieran sus síntomas con alguna medicación, o bien menospreciarían el valor de las consultas de quienes somos especialistas en medicina familiar. Es reconfortante entonces para nosotros haber podido constatar que la expectativa de recibir la prescripción de un medicamento y/o una consulta con un especialista no integran las principales expectativas de los usuarios encuestados, de manera concordante con los hallazgos comunicados por Ruiz Moral et $\mathrm{al}^{8}$. Vale destacar que en nuestra población tendría mayor valor la expectativa de recibir la indicación de realizar pruebas diagnósticas, cuando se lo compara con los resultados de estos autores, hallazgo para el cual no contamos con una explicación satisfactoria.

Entre las limitaciones de nuestro diseño podemos mencionar que la muestra seleccionada fue de conveniencia, de pequeño tamaño y limitada al mes de noviembre de 2016, que estacionalmente se comporta como un período de menos demanda al servicio de salud evaluado, por lo que los resultados obtenidos podrían no ser generalizables al resto del año. Durante el período en que se realizó la encuesta hubo un franco predominio de las consultas programadas, que suelen ser menos frecuentes durante los meses de invierno.

Por otro lado, la persona que invitó a los potenciales participantes de la investigación fue un médico de familia que ejercía su práctica clínica habitual en el mismo Centro de Salud, y si bien fueron excluidos los pacientes o los familiares de pacientes que algunas vez habían sido atendidos por él, no podemos descartar un posible sesgo de cortesía de los participantes del estudio.

Si bien existen antecedentes de la evaluación de las expectativas de los usuarios de Centros de Salud de diferentes regiones de Argentina ${ }^{17}$, estas abarcaron la valoración de lo que esperaban los pacientes del establecimiento de salud en su conjunto, y no respecto de la singularidad de una entrevista específica con un profesional de la salud evaluado durante los minutos anteriores al ingreso del usuario al consultorio en cuestión.

\section{Conclusión}

Este trabajo nos permitió conocer las principales expectativas de los pacientes adultos que consultan a un Centro de Salud de Atención Primaria situado en el conurbano bonaerense.

Hemos podido documentar que las expectativas de nuestros pacientes respecto de sus entrevistas clínicas están muy vinculadas a su componente actitudinal (predisposición a escuchar con interés sus problemas) y al de las habilidades comunicacionales del profesional de la salud (explicaciones).

\section{Referencias}

1. Sociedad Española de Medicina de Familia y Comunitaria. Tratado de Medicina de Familia y Comunitaria. 2nd ed. Casado-Vicente V, Calero-Muñoz S, Cordón-Granados F, Exquerra-Lezcano M, García-Olmos L, García-Velazco G, et al., editors. Editorial Médica Panamericana; 2012.

2. Simpson M, Buckman R, Stewart M, Maguire P, Lipkin M, Novack D. Doctor-patient communication: the Toronto consensus statement. BMJ. 1991;303(6814):1385-1392. Internet. Available from: 10.1136/bmj.303.6814.1385.

3. Cleary PD, Mcneil BJ. Patient Satisfaction as an Indicator of Quality Care. Inquiry. 1988;25(1):25-36.

4. Redondo-Martín S, Bolaños-Gallardo E, Almaraz-Gómez A, Maderuelo-Fernández JA. Percepciones y expectativas sobre la atención primaria de salud: una nueva forma de identificar mejoras en el sistema de atención. Atención Primaria. 2005;36(7):358-63. Internet. Available from: 
$10.1157 / 13080291$

5. Engel GL. The need for a new medical model: a challenge for biomedicine. Science. 1977;196(4286):129-165. Available from: 10.1126/science. 847460 .

6. Rao JK, Weinberger M, Kroenke K. Visit-specific expectations and patient-centered outcomes: a literature review. Arch Fam Med. 2000;9(10):114855. Available from: 10.1001/archfami.9.10.1148.

7. Abril-Collado RO, Cuba-Fuentes MS. Introducción A La Medicina Familiar. Acta Médica Peruana. 2013;30(1):31-36. Available from: http://www. scielo.org.pe/scielo.php?script=sci arttext\&pid=S1728-59172013000100006.

8. Ruiz-Moral R, Gavilán-Moral E, Pérula-De-Torres LA, Jaramillo-Martín I, LA. ¿Cuáles son las expectativas de los pacientes cuando acuden a una consulta de atención primaria y qué piensan los médicos sobre ellas? Rev Calid Asist. 2008;23(2):45-51. Internet. Available from: 10.1016/S1134$282 X(08) 70469-X$

9. Stewart MA. Effective physician-patient communication and health outcomes: a review. CMAJ. 1995;152(9):1423-1456. Available from: PMC1337906.

10. Kelley JM, Kraft-Todd G, Schapira L, Kossowsky J, Riess H. The Influence of the Patient-Clinician Relationship on Healthcare Outcomes: A Systematic Review and Meta-Analysis of Randomized Controlled Trials. PLoS One. 2014;9(4):e94207. Available from: 10.1371/journal.pone.0094207.

11. Kravitz RL. Measuring Patients' Expectations and Requests. Ann Intern Med. 2001;134(9 Part 2):881-889. Available from: 10.7326/0003-4819134-9_part_2-200105011-00012.

12. Nabitz U, Klazinga N, Walburg J. The EFQM excellence model: European and Dutch experiences with the EFQM approach in health care. Int J Qual Heal Care. 2000:12(3):191-202. Internet. Available from: 10.1093/intqhc/12.3.191.

13. Palacio-Lapuente F, Marquet-Palomer R, Oliver-Esteve A, Castro-Guardiola P, Bel-Reverter M, Piñol-Moreso JL. Las expectativas de los pacientes: ¿qué aspectos valoran en un centro de salud? Un estudio cualicuantitativo. Atención Primaria. 2003;31(5):307-321. Internet. Available from: $10.1016 / \mathrm{s} 0212-6567(03) 79184-7$.

14. Antoun JM, Hamadeh GN, Adib SM. What matters in the patients' decision to revisit the same primary care physician. J Med Liban. 2015;62(4):198202. Available from: $10.12816 / 0008287$.

15. Udonwa NE, Ogbonna UK. Patient-Related Factors Influencing Satisfaction in the Patient-Doctor Encounters at the General Outpatient Clinic of the University of Calabar Teaching Hospital. Int J Fam Med. 2012;2012:517027. Internet. Available from: 10.1155/2012/517027.

16. Tähepőld H, van-den Brink-Muinen A, Maaroos HI. Patient Expectations from Consultation with Family Physician. Croat Med J. 2006:47(1):148-54

17. Laurenza M. Calidad de la atención de la salud desde la perspectiva del paciente. Análisis de los resultados de una encuesta realizada en centros de atención primaria del ámbito público. Revista del ITAES. 2013;15:18-28. Available from: https://www.sadamweb.com.ar/news/2016_05Mayo/ Calidad en atencion de salud.pdf.

18. Weisbrot MA, Segalini AM, Burdisso N, Herrera AG, Grande-Ratti MF, Rezzonico MG. Análisis de Situación Integral de Salud (ASIS) de la comunidad del barrio Santa María, Bajo Boulogne, partido de San Isidro. Rev Hosp Ital B Aires. 2016;36(4):136-142. Available from: https://www1.hospitalitaliano.org.ar/multimedia/archivos/noticias_attachs/47/documentos/28649_original\%20136-42.pdf. 